\title{
SIMULATING LARGE-SCALE EVACUATION SCENARIOS IN COMMERCIAL SHOPPING DISTRICTS - METHODOLOGIES AND CASE STUDY
}

\author{
Manuel D. Rossetti \\ Qingbiao Ni \\ University of Arkansas \\ Department of Industrial Engineering \\ 4207 Bell Engineering Center \\ Fayetteville, AR 72701,USA
}

\begin{abstract}
Large-scale regional evacuation is an important component of homeland security emergency response planning; however, evacuations involving large commercial shopping areas have not been a major focus area for research initiatives. This paper presents microscopic simulation methods for modeling large scale evacuations within the context of a case study involving the evacuation of parking lots within a commercial shopping district. A base model for background traffic was constructed and validated in order to represent real traffic conditions. Six evacuation scenarios were developed and explored within simulation experiments by varying factors involving the occupancy rate of parking lots and background traffic levels. The performance of vehicles attempting to evacuate the areas was captured in terms of an evacuation risk profile involving the most problematic parking lots and areas where traffic bottlenecks are projected to occur.
\end{abstract}

\section{INTRODUCTION}

The planning of large-scale evacuation has become an important area of emphasis for emergency planners. Large-scale evacuation involves the movement of people and resources both to escape the disaster and to respond to the disaster. Such disasters include natural and man-made events (e.g. earthquakes, tsunami, wildfire, radioactive release, and terrorist attacks). In areas prone to emergency events, such as wildfire interfaces, canyon communities, large shopping malls or islands offshore, the preplanning for evacuation is necessary and crucial. For instance, more than twenty people were killed in the Oakland Hill wildfire in 1991, where most of them lost their lives within half an hour after the fire. (Church and Sexton 2002) An unprecedented devastating tsunami hit Indonesia in 2004, causing more than 16,000 deaths and thousands of homeless people, due to lack of tsunami warning systems and wellprepared evacuation plans. (Asian Development Bank 2006) Piqued by these emergency scenarios, researchers have begun to develop optimal evacuation strategies, where numerous relevant problems emerge. A central issue for evaluating evacuation strategies is how best to simulate the processes and assess the risk of emergency plans.

The analysis of evacuation situations started with static methodologies such as the bulk lane demand (Cova and Church 1997). This is often done as a preliminary analysis and because of the lack of computational resources. Since the evacuation process is dynamic with chaos and instability, rather than static, these methods have limitations. Evacuation modeling requires the details of the movement of vehicles and people, as well as the topography within the emergency planning zones, in order to realistically represent the situation. In the past twenty years, advancements in computer technology have 


\section{Rossetti and Ni}

given rise to high fidelity simulation, which make it possible to model the details and complexity of evacuation scenarios. Micro simulation, with the ability to track individual movements of resources as well as their collective behavior, has been successfully applied to various evacuation situations.

Modeling methodologies used in evacuation have received much attention in the literature. The modeling of mid to large range evacuations (e.g. neighborhoods, parking areas, large building structures, commercial districts, etc.) remains an open area of research due to the fact that more detail as to the vehicle and pedestrian movement is required. During evacuation modeling, often assumptions are made that all the vehicles or pedestrians will be released directly into the traffic flows, without considering the detailed movements within parking structures (e.g. vehicles backing out of parking spots or driveways, and the interaction with pedestrians). These assumptions are made because of the computational burden of this analysis and because adequate modeling of these processes has not yet occurred. It should be clear that the detailed modeling of how the vehicles get into the road network is necessary because of the potential effects that this time can have on emergency plans.

This paper focuses on the application of current evacuation simulation technology, addressing key modeling issues concerning the evacuation induced by emergencies from large scale commercial shopping districts with parking lots via a case study analysis. The paper first discusses some background issues related to using simulation modeling for evacuation analysis. Then, a case study is presented to illustrate the issues. Finally, conclusions concerning the case study and the use of microscopic simulation for evacuation planning is presented.

\section{BACKGROUND AND LITERATURE REVIEW}

The methodologies and the application of simulation within emergency planning have been under development for many years. Generally, there are three types of simulation approaches: micro-simulation, meso-simulation, and macro-simulation. Micro-simulation tracks the detailed movement and interaction of individual entities on the road, whereas macro-simulation models the aggregate behavior of traffic flows based on equations stemmed from analogies with fluid flows. Meso-simulation, a compromise between micro-simulation and macro-simulation, focuses on the movement of platoons of vehicles. (Pidd et al. 1996; Southworth 1991; Sheffi et al. 1982)

At the beginning of the use of simulation, only aggregate simulation was used to simulate network traffic movement because of the constraint of limited computational resources. In order to estimate the clearance time in the evacuation of the area around nuclear power plants, Sheffi et al. (1982) described a macro simulation model, NETVACI which considered the problem of dynamic traffic assignments by exploring the mathematical relationships among traffic flows, speeds, densities, and queues. NETVACI simulated the drivers' choice for certain routes based on two factors including link familiarity and myopic behavior. The probability of selecting a route was determined by the driver's preference and traffic speed in that link at each simulation interval. Southworth (1991) offered a systematic review of regional evacuation modeling. The author described an evacuation study as consisting of five separate processes: trip demand generation, evacuation departure timing, destination choice, routing assignment, and building-up of evacuation plan and analysis. Since collecting data containing population distributions involves considerable difficulty and uncertainty, especially in varying location and time of day, Southworth (1991) stated that "worst case" or "average case" evacuation scenarios could be used to approximately capture the evacuation situations.

With advances in technology, computation capability has improved the application of microsimulators. Sinuany-Stern and Stern (1993) used the simulation language (SLAM II) to perform a sensitivity analysis of the total evacuation time in a case study of a small city. They concluded that the total evacuation time was susceptible to not only the route selection mechanisms but also other traffic factors such as friction with pedestrians, intersection traversing time and population size. Church and Sexton (2002) applied microscopic simulation to a neighborhood evacuation, where multiple evacuation scenarios were developed by considering factors such as household vehicles levels, opening new road exit, and traffic control plans. Another noticeable case study is the hurricane evacuation in the Cape May 


\section{Rossetti and Ni}

County (National Center for Transportation and Industrial Productivity 2007), where emergency planning zones were partitioned into small adjacent zones for trip generation, and contra-flow lane plan was taken into consideration within the simulation modeling. Chen and Zhan (2008) developed an agent-based simulation model to simulate the collective behavior of a group by capturing the individual behavior of resources including vehicles, pedestrians and their interactions, and explored the effectiveness of evacuation strategies including simultaneous and staged evacuation.

For estimating the number of the evacuees participated in evacuation, the Miller study (Miller Consulting Inc 2001) and Nelson et al. (1989) suggested that the number of vehicles in each evacuation zone be determined by considering several factors associated with car ownership, households quantity, household participation ratio, house occupancy rate, and vehicle usage level. Cova and Johnson (2002) introduced a Poisson distribution to simulate the number of vehicles in each house at different times of the day, considering the random nature of the vehicle quantity at different times and locations.

During an evacuation, instability and perturbation may be ubiquitous in the affected region. Generally it will take some time before the public begins to mobilize within the affected region. The response time or departure time is from detecting the emergency to starting evacuation. Southworth (1991) summarized that there were four approaches used to capture the response behavior of evacuees once the hazard events happened: derivation from past evacuation data, intention survey to potential evacuees, experts evaluation, and simulation based on the diffusion of emergency warning information. Rogers and Sorensen (1991) assumed that the emergency warning dissipation process could be captured by a logistic curve or an S-shaped curve, where the cumulative percentage of warning recipients was modeled as a function of time. Cova and Johnson (2002) employed a Poisson distribution to simulate the response time of evacuees in a neighborhood.

With the development of powerful computational resources, Agent-based modeling (ABM) also called individual based modeling (IBM), has emerged for modeling system characteristics by simulating the individual behavior of the entities called agents in the system. Currently, there are a number of offthe-shelf micro-simulation packages, for example: CORSIM, VISSIM, and Paramics. CORSIM is able to simulate the traffic in local arterials and freeways, which is link-based routing without considering pedestrians. VISSIM, based on path-based routing, can simulate multiple transport modes including pedestrian with 2D and 3D presentations of results. Q-Paramics focuses on simulating the movements of people and individual vehicles, including the interaction between vehicles, and vehicles and pedestrians. It also involves the modeling of multiple transport modes with 3D presentation. (Choa et al. 2003) Based on the comparison among CORSIM, VISSIM and Paramics, the authors concluded that Paramics and VISSIM were better in simulating a specific traffic project. Krogscheepers and Kacir (2001) presented several application examples of Paramics indicating that the Paramics could perform well in simulating networks such as freeways, surface streets and dense networks. In addition, there are numerous examples where Paramics has successfully simulated traffic networks. (Chen et al. 2006; Chen and Zhan 2008; Chu et al. 2003; Ozbay et al. 2005; Satinnam et al. 2005) Paramics was used in this paper.

\section{A CASE STUDY}

In this paper, the region around Northwest Arkansas Mall and Spring Creek Centre at Fayetteville, AR, which is within the red square shown in Figure 1, was selected as the emergency planning zone for a case study. The area is a highly visited shopping region with parking lots and over 100 stores. Such an area offers a prime target for emergency events such as the release of a bio-chemical agent or a bomb attack by terrorists. Suppose an emergency event is detected to occur in a certain time during afternoon peak hours from 4:30 to 8:00 where traffic is much higher than usual. This is a worse case scenario. In such a scenario, all people (e.g. customers, staff, etc.) have to escape to safe areas within the surrounding area. The main local roads within the study region include S Thompson St, East Joyce Boulevard, Main Drive, and East Zion Road. This case study focuses on the process of how people evacuate from parking lots to arrive at safe areas as long as the emergency takes place, and how the evacuation effects traffic flows. 


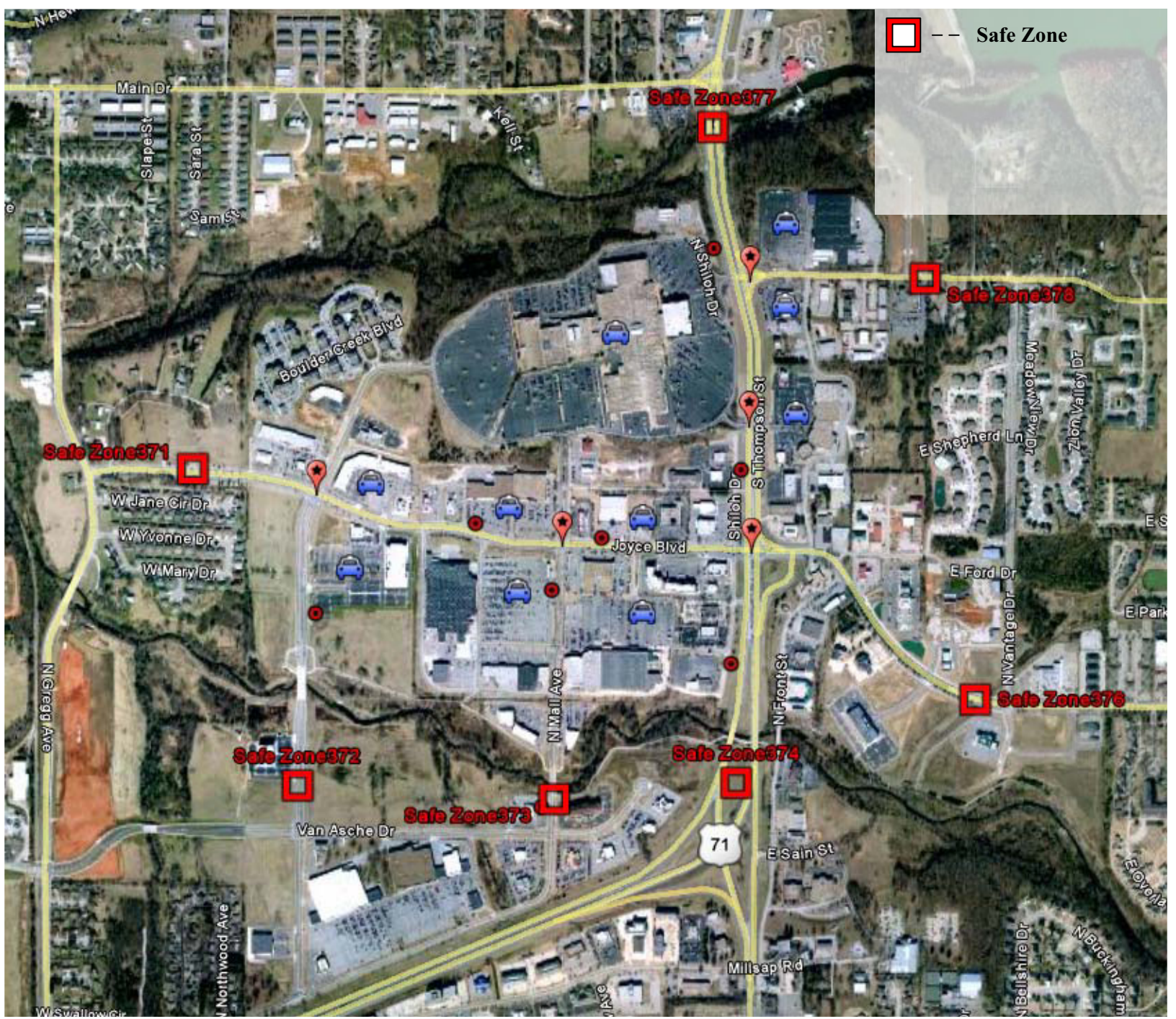

Figure 1: The Commercial Shopping Area under Study

\subsection{Key Modeling Issues}

Compared with other travel demand models, Paramics requires datasets having significantly more details for simulation model construction including route geometry data, traffic signal operation data, and parking lot layout data. There are many potential agencies (e.g. city, department of transportation, store owners, etc.) that may be helpful to acquire the necessary data. In Paramics, the simulation traffic network of the study region can be built using Converter. Converter can extract network data from several data sources (e.g. emme/2, ESRI Shape Files, MapInfo, CSV, etc.) and convert it into a basic simulation network. For the case study, ESRI shapes files from CAST (Center for Advanced Spatial Technologies) at the University of Arkansas were used as the data source to generate the road network.

Under an emergency situation, people will be desperate to leave the building, walk to their vehicles, and drive out of the parking lot into the traffic network. This process will involve the movements of vehicles and pedestrians as well as their friction. Emphasizing the movements of resources (e.g. buses, trucks, cars, and pedestrians) and their interactions during the evacuation scenarios, this paper emphasizes parking lots as the demand origination points in order to reflect the real traffic flows during the evacuation. 


\section{Rossetti and Ni}

Generally, in Paramics, a node represents an intersection, a link represents a road, and a zone built on a link can release vehicles onto network. Area zones can be used to represent residential areas, parking lots, parking rows, etc. (Quadstone, 2009) Based on the observation of parking lots, it is obvious that parking spaces are close to each other; however, Paramics cannot model this sort of traffic network in such detail. Thus, we decided to approximately model a parking lot by aggregating each parking row into several zones. Typically, there are three types of parking zones in each parking lot: the closest to the entrance of the building, the farthest, and the middle between the two outer zones. Based on aggregated parking spaces the collective behavior of each zone can be modeled; however, this ignores the details of vehicle movements contained in each zone. In addition, the method assumes that all vehicles are generated along links, to some extent which can simulate the disruption caused by the back-driving vehicles from parking space on traffic flows.

Trip generation concerns how to generate the number of evacuated vehicles from parking zones based on collected occupancy rate (OR) of parking lots, which is the ratio of the total number of vehicles currently parking at a parking lot to the capacity of the parking lot. Suppose one parking row can be divided into several parking zones. Assume there is a descending priority for drivers to park vehicles from the first row parking zones to the third row parking zones. In other words, drivers first choose to park at the parking zones closer to the entrance to the shopping buildings, then park at the middle parking zones, at lastly they decide to park at the furthest zones. Given the layout and OR of a parking lot, the average number of vehicles parking in each zone can be approximately estimated. This paper constructed the simulation models with two ORs: the current OR and $85 \%$ OR.

Given the average number of vehicles in a parking zone, this paper assumes that the number of vehicles $X$ in a parking zone is $\sim$ Poisson $(\lambda)$, where $\lambda$ represents the mean number of vehicles distributed in the parking zone. For the purposes of the case study and the short time period for the evacuation, the mean rate was assumed to be stationary; however, the rate may be different for different parking zones. In Paramics, demand files specifies the number of vehicles to travel from each origin zone to each destination zone. In this paper, we use computer programming to randomly generate the number of vehicles for each origination-destination pair and then generate different demand files for different experimental scenarios.

Destination choice modeling mainly addresses the issue of how to select safe zones in order to assign traffic demand for each origin/destination (OD). The study region can be partitioned into numerous traffic analysis zones (TAZs) based on GIS data in conjunction with population distribution information. Suppose there are limited safe zones with particular populations outside the emergency planning area, each parking zone is deemed to be one demand origin, and people (such as shoppers, pedestrians, or other staff) at each origin come from these safe zones. Therefore, it is highly possible that people choose to go to a certain safe zones (i.e. their home zone) once the emergency happens. Therefore, the assumption can be made that traffic demands from origins within the planning area to each safe zone are proportional to the population in these safe zones. For a simple base model, the traffic demand from each parking zone can be also divided equally. In this paper, seven safe zones distributed around the outside of the emergency region are designed to release background traffic and absorb evacuation traffic. For the base case modeling, we assume that the probability that a vehicle from a parking lot chooses any one of the safe zones to go is the same, that is, one seventh for a vehicle going to a safe zone.

In the departure timing step the concern is how to load traffic on road networks once the evacuation order is executed. This paper assumes that departure time includes both notification time and evacuation preparation time in an evacuation. Usually, the evacuation rate is low at the beginning, peaks gradually and later reaches a trough. Therefore it is reasonable to make an assumption that the probability density of evacuation departure events varying by time is a Poisson distribution (Cova and Johnson 2002). Based on this assumption, each evacuee can be assigned a time interval during which the departure event happens. Suppose $\mathrm{N}$ is a random variable that represents the number of the departure time intervals with $\mathrm{T}$ minute increments in the total evacuation time, and assume that $\mathrm{N}$ can be described by a Poisson distribution whose corresponding probability is (essentially) the evacuation rate during a certain time interval: 


\section{Rossetti and Ni}

$$
P_{n}=P\{N=n\}=\frac{\gamma^{n} e^{-\gamma}}{n !}, n=0,1,2 \ldots \ldots .
$$

Where $\gamma$ is the mean number of departure intervals, $P_{n}$ represents the percentage of vehicles evacuated within the time interval $\mathrm{n}$, and $\gamma^{*} \mathrm{~T}$ is the mean departure time for all vehicles.

Based on this departure model, we can determine the temporal distribution of departure vehicles. Let, $\mathrm{X}$ be the total number of vehicles generated in zones. Thus $\mathrm{X} * \mathrm{P}_{\mathrm{n}}$ represents the fraction of vehicles assigned to depart in interval $\mathrm{n}$.

\subsection{Background Traffic Model}

The base model is an initial simulation model with background traffic which involves normal traffic such as peak-hour traffic and shoppers. The objective of the base model is to represent the evacuation traffic network and simulate background traffic conditions at the beginning of an evacuation. Then, it is possible to model the interaction between evacuation traffic flow and background traffic flow on the roads. In addition, the base model can be used to assist with the validation of the simulation model by comparing the simulation results with observed data. In this research, two major assumptions are made: 1) Only major roads in the affected region will be used as evacuation routes. Background traffic flow is constrained to the main predefined roads (e.g. E Joyce Blvd, S Thompson St, and N Mall Ave) and is not allowed to use routes within parking lots. 2) Background traffic flow on the main roads of the simulation network can be represented by observed traffic flows at the observation stations shown in Figure 1.

In the paper, traffic volumes of main roads during afternoon peak hours were collected during 15 minutes intervals. In the simulation model, the background traffic is generated from a upstream safe zone along a evacuation road, and run to the downstream safe zone of the road. For validating the simulation model, the mean absolute percentage error (MAPE) between observed traffic counts and simulated traffic counts was used as a measure for goodness of fit at different observation stations.

Based on the results in Figure 2, we find that the mean absolute percentage error (MAPE) over all is $7.35 \%$ and the maximum MAPE is $9.43 \%$. Thus, we assume that the developed base model is sufficient for modeling the background traffic conditions. And hence, the assumptions made above appear reasonable and can provide a solid base for developing evacuation simulation models.
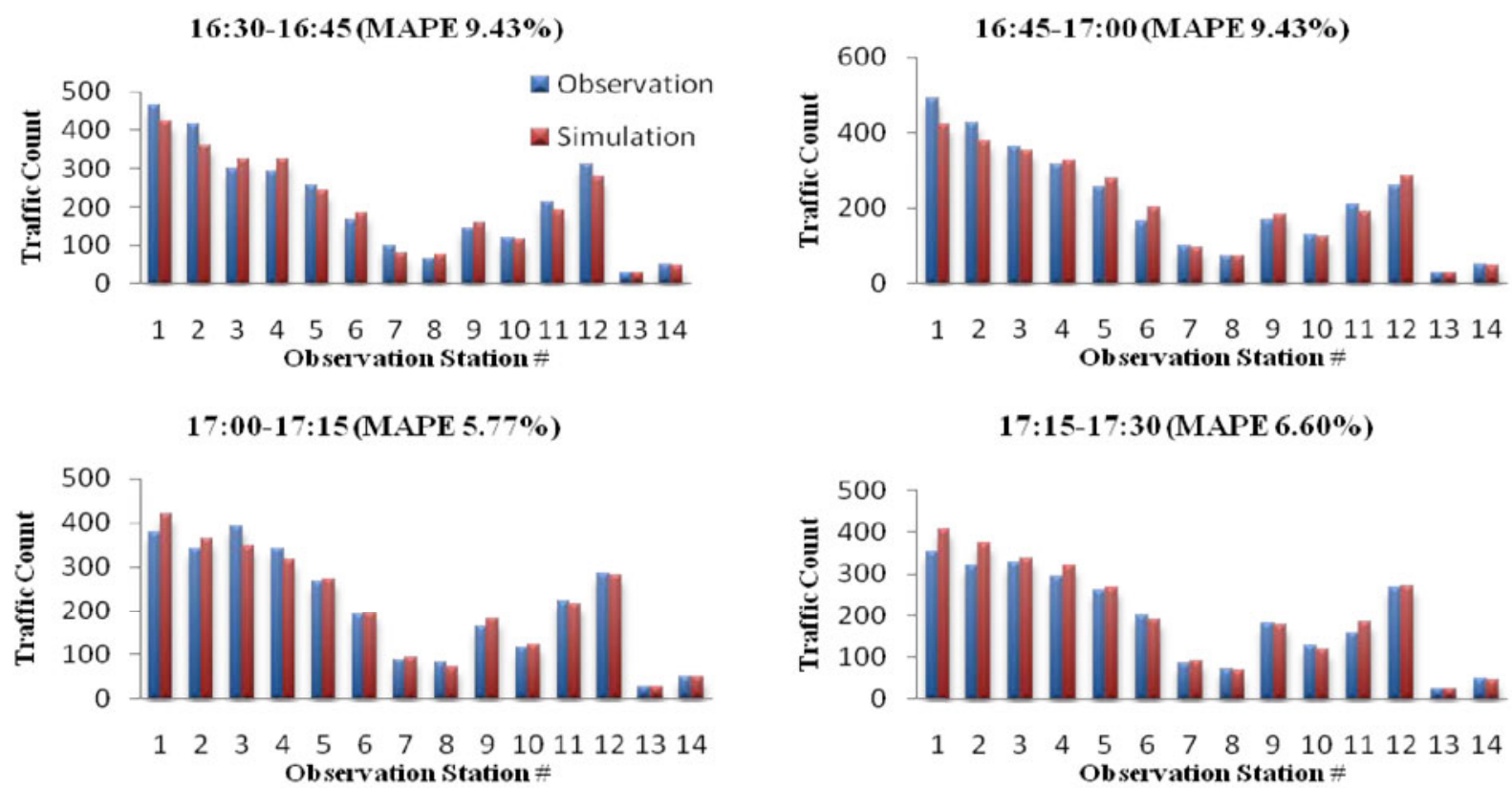

Figure 2: Comparison of Simulated and Observed Traffic Counts 


\section{Rossetti and Ni}

\subsection{Evacuation Experimentation}

The evacuation simulation model was initially constructed by loading evacuation traffic flow into the base model built in Section 3.2. According to the discussion in Section 3.1, the parking zones are assumed to be the origination of the evacuation traffic, and the number of vehicles participating in evacuation is generated by the occupancy rate and a Poisson distribution. Then, the evacuation traffic was loaded onto network by a response rate. Suppose the response rate for evacuees in the affected region is also a Poisson distribution with mean evacuation time of 15 minutes, which is the time of receiving an evacuation order, exiting a shopping building, walking to their vehicles, and beginning to drive out of parking lots. For the destination choice, the evacuated vehicles were equally distributed to seven safe zones.

Based on the constructed evacuation model, this section tests the effectiveness of the evacuation models by developing different scenarios.

\subsubsection{Evacuation Scenario Development}

Evacuation may be dependent on various factors such as the occupancy rate of the parking lots, background traffic, traffic control policies, evacuation routes assignment, response rate, etc. In this paper we only vary two factors: response rate and background traffic operation level. The origins of vehicles are parking lots in the shopping areas, and hence the occupancy rate of each parking lot will influence the total evacuation time. In this project, we consider only two occupancy rates: current occupancy rate without emergencies and an $85 \%$ occupancy rate. During evacuation, the government may guide the operation of background traffic. For example, police may stop outside traffic flow from entering the affected region. In this paper, we consider three situations concerning the background traffic:

- The background traffic stopped suddenly after 15 minutes from the beginning of the evacuation.

- The background traffic is reduced gradually after 15 minutes from the beginning of evacuation, according to a Poisson distribution.

- The background traffic remains the same during the entire the evacuation time.

Based on these factors, six evacuation scenarios were developed, which are described in more detail as follows.

- Evacuation Scenario 1 - Normal occupancy rate without evacuation and the same background traffic level during evacuation.

- Evacuation Scenario 2 - 85\% occupancy rate and the same level of background traffic

- Evacuation Scenario 3 - Normal occupancy rate without evacuation and background traffic will be turned off suddenly.

- Evacuation Scenario 4 - 85\% occupancy rate evacuation and background traffic will be turned of suddenly.

- Evacuation Scenario 5 - Normal occupancy rate with evacuation and gradually decreasing background traffic.

- Evacuation Scenario 6 - 85\% occupancy rate and gradually decreasing background traffic.

\subsubsection{Scenario Results and Analysis}

For each evacuation scenario, the simulated results were obtained by running multiple replications (at least 30) with different random seeds on the Processor module of Paramics. Simulation models were run on a desktop computer with the Dual Intel Core i7 $2.67 \mathrm{G}$ processor, 6.0G of RAM, and the 64-bit Windows Vista operating system. For one simulation replication, it generally takes over 2 minutes of real time. Thus, a scenario takes at least 1 hour to evaluate.

The total evacuation time analysis without considering particular parking lots or parking zone can provide information such as mean evacuation time (Mean ET), maximum evacuation time (Max ET), minimum evacuation time (Min ET), and cumulative density shopping area can be estimated, if the time 


\section{Rossetti and Ni}

to evacuate is given and limited. function of the number of vehicles evacuated. Based on this, the risk of evacuation faced by shoppers in the commercial

Table 1: Evacuation Time Analysis with 95\% Confidence Limits Across Scenarios

\begin{tabular}{|r|l|l|l|l|l|l|}
\hline & Scenario 1 & Scenario 2 & Scenario 3 & Scenario 4 & Scenario 5 & Scenario 6 \\
\hline Mean ET & $16.00 \pm 0.13$ & $55.35 \pm 0.19$ & $7.75 \pm 0.05$ & $28.66 \pm 0.09$ & $10.02 \pm 0.06$ & $31.88 \pm 0.10$ \\
\hline Max ET & $134.73 \pm 5.55$ & $220.40 \pm 7.23$ & $63.42 \pm 1.99$ & $104.45 \pm 2.25$ & $55.82 \pm 1.59$ & $97.02 \pm 1.99$ \\
\hline Min ET & $0.23 \pm 0.01$ & $0.23 \pm 0.02$ & $0.25 \pm 0.01$ & $0.23 \pm 0.02$ & $0.25 \pm 0.01$ & $0.23 \pm 0.02$ \\
\hline
\end{tabular}

According to Table 1, the following conclusions can be made:

- The evacuation time of model scenarios with $85 \%$ occupancy rate is greater than model scenarios with normal occupancy rates. Because the former involves much more movement of resources and easily causes much more traffic congestion in the study region. For example, it takes about 55 minutes to evacuate the whole study area in scenario 2; however, it only takes about 16 minutes in scenario 1.

- For each occupancy rate, evacuation time is the smallest in the model with background traffic turned off, and the greatest in the model with the background traffic of the same level. The evacuation time in the model with gradually reduced background traffic is in the middle. Because of less background traffic during evacuation, less interaction occurs between evacuation traffic and background traffic. For example, the evacuation time is higher for scenario 2, scenario 6, and scenario 4, where the occupancy rates are $85 \%$.

Based on the discussion in Section 3.1, the closet parking zones to the entrance of the building are defined as parking row 1 , the farthest are parking row 3 , and the middle parking zones are parking row 2 . For each parking row within a parking lot 1 shown in Figure 1, the average evacuation time is illustrated in Table 2. There is not much difference for the evacuation time for these parking rows, which may not be reasonable in reality; however, it is explainable based on the modeling. In the simulation model, the parking lot modeling was simplified, not considering the behaviors of pedestrians and vehicles in more detail. For example, the procedure to allow drivers get in their car, drive out parking lots and escape to safe zones was considered; however, the process of how drivers get out of buildings and find their cars in parking lots, which can affect evacuation time greatly was not modeled. Without considering the detailed movements in parking lots, the evacuation time for different parking rows may not be very different. This suggests that the detailed modeling of the movements within parking lots during an evacuation is an important area for future research. The authors are unaware of any commercial off the shelf traffic modeling programs that can effectively model this situation.

Table 2: Average Evacuation Time with 95\% Confidence Limits For Parking Rows Across Scenarios

\begin{tabular}{|l|l|l|l|l|l|l|}
\hline & Scenario 1 & Scenario 2 & Scenario 3 & Scenario 4 & Scenario 5 & Scenario 6 \\
\hline Parking row 1 & $17.97 \pm 0.36$ & $46.87 \pm 0.67$ & $10.79 \pm 0.18$ & $31.47 \pm 0.39$ & $12.63 \pm 0.21$ & $33.52 \pm 0.44$ \\
\hline Parking row 2 & $17.04 \pm 0.60$ & $45.40 \pm 0.68$ & $10.48 \pm 0.28$ & $30.81 \pm 0.40$ & $12.63 \pm 0.35$ & $32.48 \pm 0.46$ \\
\hline Parking row 3 & $33.27 \pm 16.70$ & $45.62 \pm 0.83$ & $18.33 \pm 5.14$ & $30.55 \pm 0.48$ & $17.98 \pm 14.42$ & $33.75 \pm 0.56$ \\
\hline
\end{tabular}

The evacuation time distribution for different destination measures the time taken for a vehicle to get to a safe zone. The average evacuation time for each destination in different scenarios is provided in Table 3. Based on the table, safe zones 371 and 372 are the best safe zones for evacuees, which require the least evacuation time. According to the map of study region and simulation runs, 371 and 372 are not only closer to shopping areas but also experience lower traffic congestion.

Evacuation always occurs along with massive movements of resources and people. It is inevitable that traffic congestion happens. Therefore, the identification of traffic bottlenecks is an important aspect in the 


\section{Rossetti and Ni}

emergency planning. Take evacuation situation at 16:50 in the scenario 2, for example. As shown in Figure 3, yellow circles represent the traffic congestion. Most of the traffic gridlock happens at the exits connecting the main roads inside of the parking lots. The major congestion occurs at road A, road B, road $\mathrm{C}$ and road $\mathrm{D}$. The traffic congestion at road $\mathrm{A}$ is the most important for reducing entire evacuation traffic, because it causes serious traffic congestion at the intersection of N College Ave and E Joyce Blvd, which in turn causes the traffic congestion at other locations.

Table 3: Average Evacuation Time with 95\% Confidence Limits For Destinations Across Scenarios

\begin{tabular}{|r|llllll|}
\hline Destination & Scenario 1 & Scenario 2 & Scenario 3 & Scenario 4 & Scenario 5 & Scenario 6 \\
\hline 371 & $9.00 \pm 0.24$ & $48.39 \pm 0.47$ & $\mathbf{3 . 9 0} \pm 0.07$ & $\mathbf{2 4 . 8 0} \pm 0.24$ & $5.70 \pm 0.11$ & $\mathbf{2 7 . 8 0} \pm 0.25$ \\
372 & $\mathbf{8 . 1 8} \pm 0.20$ & $\mathbf{4 7 . 6 5} \pm 0.47$ & $3.95 \pm 0.07$ & $25.13 \pm 0.24$ & $\mathbf{5 . 6 4} \pm 0.10$ & $28.08 \pm 0.25$ \\
373 & $15.41 \pm 0.32$ & $54.12 \pm 0.47$ & $7.31 \pm 0.11$ & $27.84 \pm 0.22$ & $9.48 \pm 0.14$ & $31.18 \pm 0.24$ \\
374 & $19.94 \pm 0.35$ & $59.28 \pm 0.49$ & $9.09 \pm 0.14$ & $30.25 \pm 0.24$ & $11.89 \pm 0.17$ & $33.57 \pm 0.25$ \\
376 & $20.49 \pm 0.36$ & $62.06 \pm 0.50$ & $9.85 \pm 0.15$ & $31.08 \pm 0.24$ & $12.82 \pm 0.18$ & $34.59 \pm 0.25$ \\
377 & $19.58 \pm 0.38$ & $56.92 \pm 0.51$ & $10.20 \pm 0.15$ & $30.70 \pm 0.25$ & $12.47 \pm 0.19$ & $33.87 \pm 0.26$ \\
378 & $19.32 \pm 0.37$ & $59.09 \pm 0.50$ & $9.92 \pm 0.14$ & $30.75 \pm 0.24$ & $12.25 \pm 0.18$ & $34.15 \pm 0.26$ \\
\hline
\end{tabular}

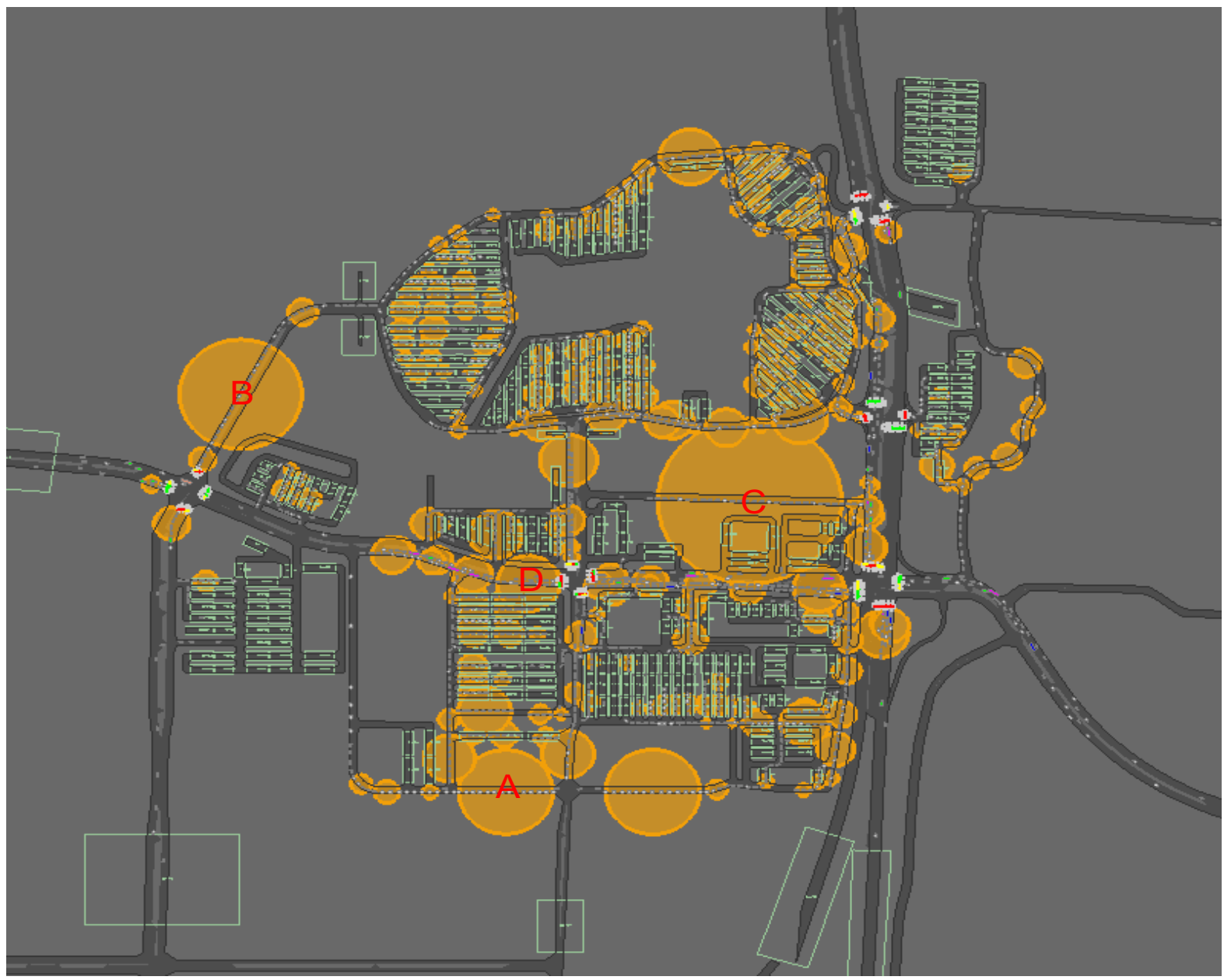

Figure 3: Traffic Bottlenecks in Study Region in Scenario 2 


\section{Rossetti and Ni}

\section{SUMMARY AND CONCLUSIONS}

In this paper, we have addressed key modeling issues for the evacuation of a large shopping district and have successfully performed a case study in the region around the Northwest Arkansas Mall at Fayetteville, AR; however, we have encountered many challenges and lessons learned concerning modeling issues and there are still several areas open for future studies. Compared with current traffic simulation packages, Paramics is one of the most cutting edge micro-simulation software programs in the world. In our research, we therefore selected Paramics for our evacuation modeling; however, many modeling issues occurred during the modeling process.

- Current traffic simulation packages have inadequate modeling capabilities for serious evacuation studies. The packages cannot easily incorporate stochastic modeling and experimentation. Trip demand files and profiles files cannot be generated by the simulator. Scenarios have to be generated externally imported from other packages. This makes experimentation on the models difficult. In addition, the packages are generally not setup for statistical experimentation methods.

- The packages cannot model the detailed dynamics within a parking lot. Micro-simulators cannot model such details as the individual parking space; therefore, we have to use a simplified method by aggregating parking spaces into parking zones, ignoring the details inside parking zones. This makes it difficult to include an important evacuation process in the modeling.

- The pedestrian modeling tools are in their infancy. One of the modeling issues for the evacuation in shopping areas is to model the interaction between vehicles and pedestrians in the parking lots. We desire to model the panic-stricken situation that shoppers escape from buildings, drive their cars and leave parking lots to safe zones. In our research, we cannot model the process that pedestrians desperately walking around to find their cars and leave with their cars, since pedestrians are only allowed to travel on predefined route in many micro-simulation packages.

- Data collection is a serious challenge. Micro-simulators require detailed data in the study region; therefore much effort has to be made in acquiring data. For example, consider our study region. Much time was spent on data collection including: counting parking lot occupancy, road volumes, network structure, road control, parking lot structure, demand patterns, parking lot patterns, evacuation initiation, etc.

- Model calibration is a serious challenge and a well established problem with the use of microscopic simulation. Not only does it take significant time to calibrate a single evacuation model, but realistic modeling must calibrate multiple evacuation models during experimentation. At present, trial and error is the major method used for model calibration, which is not an acceptable long term approach.

- Although analysis tools are available in the micro-simulation packages to facilitate simulation results analysis, many packages require post-processing of extensive data sets. In some instances custom programming is necessary to access the desires statistics of evacuation studies. For example, we estimate the evacuation risk as a function of time by using external statistical packages. These statistics are not readily available within many commercial off the shelf software packages. In our research, the Analyser of Paramics was unable to extract our desired data results such as traffic bottleneck locations, because of the necessity of having many zones represent parking locations.

- In the model scenario development, we only consider two factors: occupancy rate of a parking lot and background traffic levels. In fact, a number of other factors can affect the effectiveness of evacuation such as traffic operations, response rate, evacuation sequence, etc. For example, evacuation time may be much different if a staged evacuation policy is used, rather than simultaneous evacuation. The analysis of these issues is part of on-going research efforts.

- As discussed in previous sections, the modeling of parking lots is crucial to the overall evacuation of shopping areas. It not only includes modeling the individual parking spaces as trip generation locations, but also modeling the interaction between pedestrians and vehicles in parking lots at the 


\section{Rossetti and Ni}

beginning of an evacuation. This is a deficiency in many simulation packages. The more details we model within parking lots, the more accurate the result we will be able to obtain.

- Under evacuation, pedestrians may distribute in the affected regions not only in the parking lots but also on the evacuation routes. The friction between pedestrians and evacuation traffic is inevitable. Therefore the modeling of pedestrians is also an important aspect in evacuation modeling. For example, in order to simulate the movement of pedestrians, we need to determine the distribution of pedestrians at different time of the day, movement mode (e.g. walking or running), and their route choice under an evacuation situation.

On-going and future work is planned to address some of these items. In addition, future work is planned to better understand how the layout of the parking lot effects the evacuation time.

\section{ACKNOWLEGMENT}

This material is based upon work supported by the U.S. Department of Homeland Security under Grant Award Number 2008-ST-061-TS003. The views and conclusions contained in this document are those of the authors and should not be interpreted as necessarily representing the official policies, either expressed or implied, of the U.S. Department of Homeland Security.

\section{REFERENCES}

Asian Development Bank. 2006. Indonesia: Tsunami Summary, Available via < http://www.adb.org/Media/Articles/2005/6618_tsunami_impact_Indonesia/default.asp?> [accessed May 3, 2009]

Chen, X., and F. B. Zhan. 2008. Agent-Based Modeling and Simulation of Urban Evacuation: Relative Effectiveness of Simultaneous and Staged Evacuation Strategies. Journal of the Operational Research Society 59:25-53.

Chen, X., J. W. Meaker, and F.B. Zhan. 2006. Agent-based modeling and analysis of hurricane evacuation procedures for the Florida Keys. Natural Hazards 38:321-338.

Choa, F., R. T. Milam, and D. Stanek. 2003. CORSSIM, PARAMICS AND VISSIM: What the Manuals Never Told You. In Proceeds of 9th TRB Conference on Planning Methods. Baton Rouge, La.

Chu, L., H. X. Liu, J. S. Oh, and W. Recker. 2004. A Calibration Procedure for Microscopic Traffic Simulation. In Proceedings of TRB 2004 Annual meeting, USA

Church, R., and R. Sexton. 2002. Modeling Small Area Evacuation: Can Existing Transportation Infrastructure Impede Public Safety?. Caltrans Testbed Center for Interoperability Task Order 3021 Report, Vehicle Intelligence \& Transportation Analysis Laboratory, University of California, Santa Barbara.

Cova, T. J., and J. P. Johnson,. 2002. Microsimulation of neighborhood evacuations in the urban-wildland interface. Environment and Planning A 34: 2211-2229.

Cova, T. J., and R. Church. 1997. Modeling community evacuation vulnerability using GIS. International Journal of Geographic Information Science 11:763-784.

Krogscheepers, C., and K. Kacir. 2001. Latest trends in micro simulation: an application of the Paramics model. 20th South African Transport Conference, South Africa. BKS (Pty) Ltd, 222 Durban Road, Bellville, RSA.

Miller Consulting Inc. 2001. Florida Keys hurricane evacuation report. Contract No. C7391, Florida Department of Transportation, Miami, Florida.

National Center for Transportation and Industrial Productivity. 2007. Cape May County Hurricane Evacuation and Elevation Study Extension. Final report, New Jersey Institute of Technology, Newark, NJ 07102-1983. 


\section{Rossetti and Ni}

Nelson, C. E., M. D. Coovert, A. Kurtz, B. Fritzche, C. Crumley, and A. Powell. 1989. Models of hurricane evacuation behavior. Department of Psychology, University of SouthFlorida, Tampa, FL.

Ozbay, K., S. Mudigonda, and B.O. Bartin. 2005. Development and Calibration of an Integrated Freeway and Toll Plaza Model for New Jersey Turnpike using Paramics Microscopic Simulation Tool. In Proceedings of the 8th International IEEE Conference on Intelligent Transportation Systems. Vienna, Austria.

Pidd, M., F. N. de Silva, and R.W. Eglese. 1996. A Simulation Model for Emergency Evacuation. European Journal of Operational Research 90:413-419.

Qudstone. 2009. Quadstone Paramics V6.5.3 modeller user guide. Edinburgh, UK.

Rogers G. O., and J. H. Sorensen. 1991. Diffusion of emergency warning: comparing empirical and simulation results. In Risk Analysis Prospects and Opportunitie, E. C. Zerros, K. E. Knox, L. Abramson, R. Coppock. Plenum Press, New York.

Satinnam, T., R. Oshima, A. Fukuda, and P. Klungboonkrong. 2005. Comprehensive Transportation Policy Development Using Traffic Simulation Technique for Khon Kaen City, Thailand. In Proceedings of the Eastern Asia Society for Transportation Studies 5:2352-2364.

Sheffi, Y., H. Mahmassani, and W. B. Powell. 1982. A transportation network evacuation model. Transportation Research A 16: 209-218.

Sinuany-Stern, Z., and E. Stern. 1993. Simulating the evacuation of a small city: The effects of traffic factors. Socio-Economic Planning Sciences 27: 97-108.

Southworth, F. 1991. Regional evacuation modeling: a state-of-the-art review. ORNL/TM- 11740, Oak Ridge National Laboratory, PO Box 2008, Oak Ridge, TN37831

\section{AUTHOR BIOGRAPHIES}

MANUEL D. ROSSETTI is a Professor in the Industrial Engineering Department at the University of Arkansas. He received his Ph.D. in Industrial and Systems Engineering from The Ohio State University. $\mathrm{He}$ serves as an Associate Editor for the International Journal of Modeling and Simulation and is active in IIE, INFORMS, and ASEE. He was a WSC proceedings editor in 2004 and a co-editor for the WSC 2009 conference. He is also author of the textbook, Simulation Modeling and Arena published by John Wiley \& Sons. His email is <rossetti@uark.edu>

QINGBIAO NI is an graduate student in the Industrial Engineering Department at the University of Arkansas. He received a Bachelors of Science in Industrial Engineering from Zhejiang University, Hangzhou, China. His research interests include the modeling, analysis and optimization of manufacturing and logistics systems using techniques from computer simulation, operation research, and statistics. His email is <nqb225 g gmail.com> 